\title{
Red fluorescent protein structure
}

Researchers have reported the atomic resolution structure of DsRed (Nat. Struct. Biol. 7, 1133-1138, 2000), a red fluorescing homolog of green fluorescent protein (GFP) originally isolated from Discosoma coral (Nat. Biotechnol. 17, 969-973, 1999). Unlike monomeric GFP, four monomers of DsRed self associate at two chemically distinct surfaces to form a tight tetramer in solution and in the crystal structure. According to Mark Wall, the paper's first author, each subunit shows GFP's characteristic paint in a can fold of eleven $\beta$ strands surrounding a central $\alpha$-helix, which bears the chro-

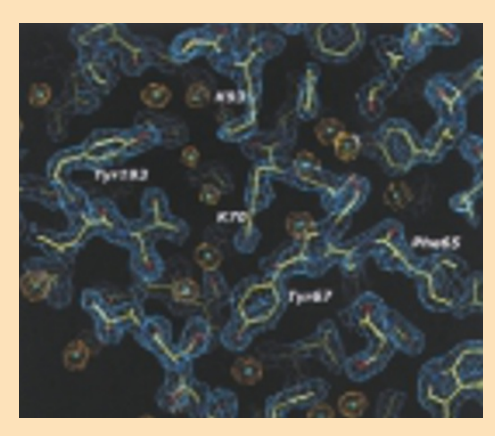
mophore generated by cyclization and oxidation of residues Gln66-Tyr67-Gly68. Remarkably, an oxidized bond flattens the main chain of Gln66 extending $\pi$ bonding of the chromophore and contributing to the far red emission spectrum of the protein. Also, cis peptide bond formation between Phe65 and Gln66 in the chromophore appears important to position the oxidized main chain of Gln66 in-plane with the chromophore and is likely to slow the maturation of DsRed's fluorescence emission spectrum from faint to intense red, which can take up to $48 \mathrm{~h}$. While optimizing DsRed so that fluorescence maturation is faster may be challenging, Wall and coauthors believe the "structural information will allow protein engineers to alter chromophore tuning and oligomeization of DsRed," making the protein more applicable for use in single and double fluorescent-label studies.

\section{Defensin stimulant}

By screening microbial extracts for products that stimulate innate host defense mechanisms, scientists at Magainin Pharmaceuticals (Plymouth Meeting, PA) have determined that L-isoleucine and several of its analogs can induce the expression of $\beta$-defensins (PNAS 97, 12723-12728, 2000). Isoleucine, which is nontoxic and can be given as a food additive, is now being tested as an alternative to antibiotics in farm animals. Defensins, bactericidal proteins secreted by mucosal epithelial cells, have been studied extensively as potential tools for combating bacterial infections, but the new work demonstrates that defensin production can be induced in cultured cells using isoleucine, an essential amino acid in the human diet that has already been proven safe. Indeed, senior author Michael Zasloff says that "with several large animal feed producers we have been evaluating the utility of isoleucine as a feed additive...in the poultry industry." The amino acid may also be incorporated into a new antimicrobial vaginal gel being developed by the company. In both cases, the researchers hope that inducing defensins prophylactically will prevent pathogenic bacteria from becoming established in the host, reducing the need for subsequent antibiotic treatment.

$A D$

Research News Briefs written by Aaron Bouchie, Alan Dove, and Andrew Marshall.

\section{Stem cells hunt tumors}

Adding to the list of potential uses for neural stem cells, scientists at Harvard University (Boston, MA) and Layton Bioscience (Sunnyvale, CA) have found that stem cells can migrate throughout the brain and preferentially juxtapose themselves to metastasizing tumor cells. Experiments in rats demonstrated that these stem cells can be used to deliver therapeutic molecules to target tumor cells with impressive accuracy. A major barrier to the treatment of brain tumors has been tumor cells' tendency to migrate widely through the brain, making them inaccessible to traditional therapies. In the new work, reported in Proc. Natl. Acad. Sci. USA (97, 12846-12851, 2000), researchers demonstrate that neural stem cells distribute themselves throughout experimentally induced gliomas in adult rats and also "chase down" migrating tumor cells elsewhere in the brain. When the stem cells are engineered to express cytosine deaminase, they can convert a nontoxic "prodrug" into an active chemotherapeutic agent, killing adjacent tumor cells. Evan Snyder, a senior author on the paper, asserts that this type of treatment "might be one of the first applications of neural stem cells for a true disease." He suggests that clinical trials using this approach could be as close as "two years away."

\section{Chromosomal checkup}

The $70 \%$ failure rate of in vitro fertilization (IVF) treatments for human couples is thought to be the result of natural embryonic chromosomal abnormalities. UK researchers have attempted to tackle the problem by employing whole genome amplification (WGA) coupled with comparative genomic hybridization (CGH), providing the first comprehensive assessment of chromosome copy number in human embryos. CGH reveals the copy number of every chromosome segment $>10 \mathrm{Mb}$ in size, but requires 10,000 times the DNA of a single cell. The approach, described in Molecular Human Reproduction (6, 10551062, 2000), employs degenerate PCR to amplify sequences of interest in cells (blastomeres) from 3-day old embryos, and then single-cell CGH to detect genome wide amplifications/deletions using an equimolar mixture of normal and test cells. Not surprisingly, nine of the embryos contained cells with likely fatal chromosomal aberrations, ranging from multiple aneuploidy to partial deletions and their combinations. According to Wells, the next goal is "to reduce the length of time that the procedure requires and to optimize the protocol for clinical application."

$A B$

\section{Artificial blood}

The use of recombinant hemoglobin as an oxygen carrier in artificial blood substitutes has had limited success due to the toxicity of the free molecule. Now a team of researchers has produced a recombinant hemoglobin that appears to solve many of these problems and may eventually form the basis for a novel class of artificial blood products. Recombinant hemoglobin in a blood substitute needs to have a lower affinity for oxygen than natural hemoglobin, as it has to function in the absence of effector molecules found inside red blood cells. Unfortunately, hemoglobin variants with low oxygen affinity tend to be less stable, and recombinant hemoglobin also tends to produce hypertension by interfering with the nitric oxide signaling pathway. In the new work (Biochemistry 39, in press, 2000), senior author Chien Ho and his colleagues identified two amino acid changes that lead to low oxygen affinity, high stability, and resistance to nitric oxide-induced oxidation in hemoglobin. "[The molecule] shows great potential as a successful oxygen carrier, and is something that could realistically be used in people one day," Before it reaches humans, the new hemoglobin will need to be produced on a larger scale, possibly in transgenic animals. 